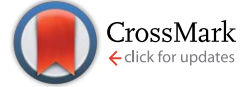

Cite this: RSC Adv., 2017, 7, 1118

Received 25th October 2016 Accepted 28th November 2016

DOI: $10.1039 / c 6 r a 25826 c$

www.rsc.org/advances

\section{Electrochemical design of a new nanosensor based on cobalt nanoparticles, chitosan and MWCNT for the determination of daclatasvir: a hepatitis C antiviral drug}

\begin{abstract}
Shereen M. Azab*a and Amany M. Fekry ${ }^{b}$
Daclatasvir (DAC) is listed on the World Health Organization's list of essential medicines needed in a basic health system, therefore, electrochemical and impedance spectroscopic methods are necessary to obtain information about its mechanism using a carbon paste electrode (CPE) modified with chitosan (Cs)/multiwalled carbon nanotubes (CNT) and cobalt nanoparticles (CoNps). The simultaneous determination of DAC with the hepatitis B antiviral drug entecavir (ENT) was also investigated by differential pulse voltammetry in universal buffer $\mathrm{pH}$ 2.0. The experimental results specify a linear relation between the DAC peak current and its concentration in the range from $1.0 \mathrm{nM}$ to $12 \mu \mathrm{M}$, leading to a detection limit of $8.82 \times 10^{-10} \mathrm{M}$. Finally, this novel sensor was successfully used to determine DAC in human biological fluids such as urine, blood serum and in pharmaceutical formulations.
\end{abstract}

\section{Introduction}

The introduction of the direct-acting antiviral drug; daclatasvir (DAC) is altering the treatment selections for hepatitis $\mathrm{C}$ virus (HCV). DAC is used in the treatment of chronic hepatitis C genotype 3 infections, it hinders the HCV nonstructural protein NS5A..$^{1,2}$ Modern research proposes that it targets two steps of the viral replication route, allowing fast decline of HCV RNA. ${ }^{1}$ Chitosan (Cs) is a natural cationic biopolymer of $\beta-(1 \rightarrow 4)$ linked 2-amino-deoxy-D-glucopyranese that has both hydroxyl groups which might be oxidized to $-\mathrm{CHO}$ or $-\mathrm{COOH}$, and amino groups that provides lone pair electrons for metal ions. Cs can be modified easily possessing adhesion properties, ${ }^{3}$ so it improves hydrophilicity and biocompatibility via covalent grafting techniques. Cs has been widely used in sensor preparations since it has affinity toward metal ions. Chitosan films can be functionalized with other substances such as polymers, nanostructures, biomolecules, or dyes to improve the film's mechanical properties. Chitosan has high adsorption properties in its matrix. ${ }^{24}$ Chitosan modified electrodes were used for the determination of heavy metals. ${ }^{4-6}$ Conducting polymer sensors have been used successfully for drug determination to improve selectivity of analysis. ${ }^{7,8}$ CNTs are porous materials that offer high effective surface area, mass transfer and catalysis due to their mechanical strength, electrical conductivity and

\footnotetext{
${ }^{a}$ Pharmaceutical Chemistry Dept., National Organization for Drug Control and Research [NODCAR], 6 Abu Hazem Street, Pyramids Ave, P. O. Box 29, Giza, Egypt. E-mail: sheryspecial@yahoo.com; Tel: +20 201225591629

${ }^{b}$ Chemistry Department, Faculty of Science, Cairo University, Giza-12613, Egypt
}

chemical stability. A decrease in over-potential can be detected with CNTs producing a more reversible voltammetry., ${ }^{9,10}$ Thus, CNTs can powerfully enhance electron transfer processes. Combining chitosan with magnetic nanoparticles is an alternative approach to reduce the adverse effects of nanoparticles. ${ }^{11}$

Recently, cobalt nanoparticles have been used in medical applications because of their good magnetic properties and their unlimited properties on proton relaxation. ${ }^{12}$ They can enter the human cells very rapidly, ${ }^{13}$ which has drawn the interest of researchers toward it. The co-existence of chitosan, CNTs and Co nanoparticles creates a negative charge which is favorable for the further immobilization of biomolecules that are positively charged. Up to date, only few articles concerning the determination of DAC using liquid chromatographic methods were reported. ${ }^{14-17}$ To our knowledge, there no electrochemical method fabricated for the determination DAC either alone or in the presence of interfering substances. In the present article, a novel modified electrode was developed for the first time by electro-polymerization of cobalt nanoparticles on the surface of CPE mixed with chitosan/CNT to be used for the electrochemical determination of daclatasvir either in commercial pharmaceutical samples or in real samples.

\section{Experimental}

\subsection{Materials and equipment}

Daclatasvir (DAC) and Daktavira tablets were provided from the European Egyptian Pharmaceutical Industries, Egypt, chitosan was purchased from sigma and the multiwalled carbon nanotubes was purchased from sigma and was immersed in 
concentrated sulfuric and nitric acid (3:1) for twelve hours then was washed with distilled water and neutralized to $\mathrm{pH}$ 7.0, and dried at $120{ }^{\circ} \mathrm{C}$. Universal buffer (B-R buffer) of $\mathrm{pH}$ 2-11 $\left(\mathrm{CH}_{3} \mathrm{COOH}+\mathrm{H}_{3} \mathrm{BO}_{3}+\mathrm{H}_{3} \mathrm{PO}_{4}\right)$, was used as the supporting electrolyte, then $0.2 \mathrm{M} \mathrm{NaOH}$ was used to gain the desired $\mathrm{pH}$ value. All voltammetric and impedance measurements were done by EC-Lab SP 150 Potentiostat electrochemical workstation. A typical three-electrode cell of $25 \mathrm{~mL}$ volume was used: a platinum rod as a counter electrode (CE), saturated calomel electrode (SCE) as a reference electrode (RE) and CPE/Cs/CNTCo as the working electrode (WE). For electrochemical impedance spectroscopic (EIS) measurements, the excitation ac signal has amplitude of $10 \mathrm{mV}$ and a frequency range from $0.1 \mathrm{~Hz}$ to $100 \mathrm{kHz}$. Thales software was used to analyse the experimental spectra.

SEM measurements were performed by SEM Model Quanta 250 FEG (Field Emission Gun) with EDX unit, with accelerating voltage $30 \mathrm{kV}$, magnification 14 up to 1000000 and resolution for Gun.1n (FEI company, Netherlands), FT-IR spectrum were performed by Agilent technologies Cary 630 and TEM images were performed by JEM-2100 (high-resolution transmission electron microscope) (HRTEM).

\subsection{Preparation of different sensors}

Carbon paste electrode (CPE) was prepared by mixing graphite powder $(0.5 \mathrm{~g})$ with drops of nujol oil in a glassy mortar. CPE modified with chitosan and CNTs was prepared by dissolving $100 \mathrm{mg}$ chitosan in $10 \mathrm{~mL}$ of $1.0 \% \mathrm{v} / \mathrm{v}$ acetic acid solution and stirred for $3 \mathrm{~h}$ at room temperature until complete dissolution then $1.0 \% \mathrm{w} / \mathrm{w}$ CNT was dispersed in the paste and vigorous sonication was applied to form $\mathrm{CPE} / \mathrm{Cs} / \mathrm{CNT}$. Then CPE/Cs/CNT electrode was deep into $1.0 \mathrm{mM} \mathrm{CoSO}_{4}$ solution, and electrodeposition of Co-nanoparticles was carried out at $-0.8 \mathrm{~V}$ for $6 \mathrm{~s}$, followed by gentle washing with deionized water to form the $\mathrm{CPE} / \mathrm{Cs} / \mathrm{CNT}-\mathrm{Co}$ sensor.

\subsection{Validation in pharmaceutical and biological samples}

The commercial pharmaceutical Daktavira (60 mg per tablet) were powdered and dissolved in deionized water and filtered to achieve $1 \mathrm{mM}$ DA then DPV were recorded. The urine and serum samples were taken from healthy volunteer, aliquot volume of urine and serum samples from healthy volunteers were diluted respectively, with $\mathrm{B}-\mathrm{R}$ buffer $\mathrm{pH}$ 2.0. The samples were centrifuged for $5 \mathrm{~min}$ at $5000 \mathrm{rpm}$ and treated with $0.7 \mathrm{~mL}$ acetonitrile as a precipitating agent to get rid of protein residues, and then the supernatant was taken carefully. All experiments were performed in compliance with the ethical guidelines for biomedical research involving human subjects', and approved by the Egyptian Network of Research Ethics Committees. Informed consents were obtained from human participants of this study.

\section{Results and discussion}

\subsection{Methodology characteristics}

3.1.1. Electrochemistry of CPE/Cs/CNT-Co. The first cyclic voltammetric behavior of $1.0 \mathrm{mM}$ DAC in $\mathrm{B}-\mathrm{R}$ buffer $\mathrm{pH} 2.0$ at scan rate $100 \mathrm{mV} \mathrm{s}^{-1}$ using bare CPE $(16 \mu \mathrm{A})$, yields one well defined oxidation peak due to the oxidation of the two pyrrolidine groups (Scheme 1). Also the presence of Cs and CNTs (CPE/ $\mathrm{Cs} / \mathrm{CNT}$ ) in the matrices of carbon paste or Co nanoparticles on the surface of the electrode (CPE/Co) causes the current to increase to $37 \mu \mathrm{A}$ and $58 \mu \mathrm{A}$, respectively. Since the presence of Cs/CNT can increases the electroactive surface area of the electrode due to the bio-adhesive character of Cs and the $\mathrm{sp}^{2}$ hybridization of CNTs, resulting in the $\pi-\pi$ interactions between adjacent layers of CNTs forming more structural defects $^{18}$ that attract more Co nanoparticles on the surface giving a larger surface area $\mathrm{CPE} / \mathrm{Cs} / \mathrm{CNT}-\mathrm{Co}$ sensor, which increases the electrode kinetics and improves the electrocatalytic effect of DAC to reach $79 \mu \mathrm{A}$ (Scheme 2).

The cyclic voltammograms of the four electrodes show the enhancement effect of the electrochemical oxidation peaks of DAC upon the addition of the modifiers in the order of CPE/Cs/ $\mathrm{CNT}-\mathrm{Co}>\mathrm{CPE} / \mathrm{Co}>\mathrm{CPE} / \mathrm{Cs} / \mathrm{CNT}>$ bare $\mathrm{CPE}$ (Fig. 1). The enhancement in current response is due to the increase in the surface area owing to the extensive cross-linkage formed between carboxyl $(-\mathrm{COOH})$ groups on CNT with hydroxyl $(-\mathrm{OH})$ and amino $\left(-\mathrm{NH}_{2}\right)$ groups in the chitosan forming $\mathrm{CPE} / \mathrm{Cs} / \mathrm{CNT}$ surface that traps DAC within the porous nanocomposite. ${ }^{19,20} \mathrm{It}$ is also observed that the potential of DAC anodic peak decreases from $1080 \mathrm{mV}$ in case of using bare CPE to $991 \mathrm{mV}$ when CPE/ Cs/CNT-Co was used. Also the electrodeposition time of CoNps was an important issue in the fabrication of this sensor so the relation between the deposition time (2-12 s) and the current response of DAC after the electrodeposition of cobalt nanoparticles (inset) was studied, the result shows that $6 \mathrm{~s}$ were enough to give the highest oxidation response of DAC.<smiles>[R]c1ncc(-c2ccc(-c3ccc(-c4cnc([R])[nH]4)cc3)cc2)[nH]1</smiles><smiles>COC(=O)N[C@H](C(=O)N1CCCC1)C(C)C</smiles> 


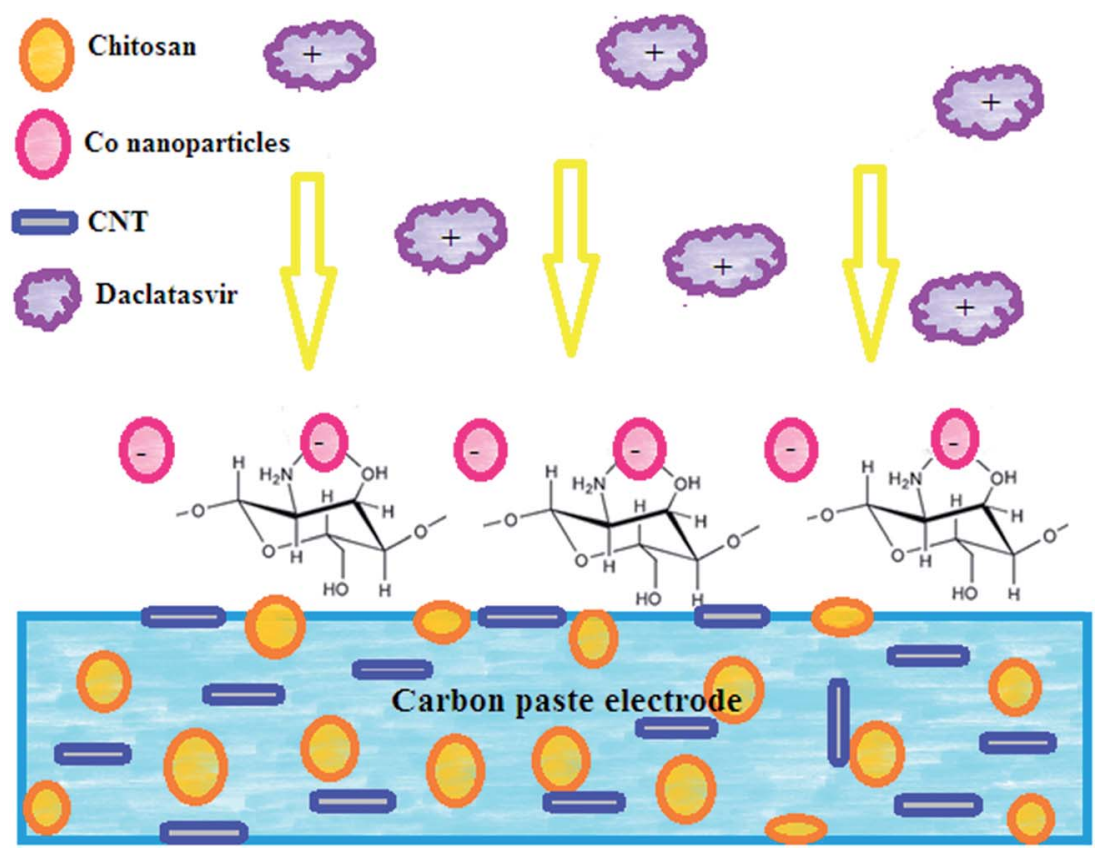

Scheme 2

\subsubsection{Surface characterization}

3.1.2.1. SEM. The surface morphology of (A) bare CPE, (B) $\mathrm{CPE} / \mathrm{Cs} / \mathrm{CNT}$ and (C) CPE/Cs/CNT-Co were represented by SEM respectively in Fig. 2. Bare CPE appears like a dark and smooth surface, while CPE/Cs/CNT shows brittle, rough chitosan bead which act as conductive cores that facilitate the transfer of electrons, and CNTs appears as unbundled, aligned, randomly

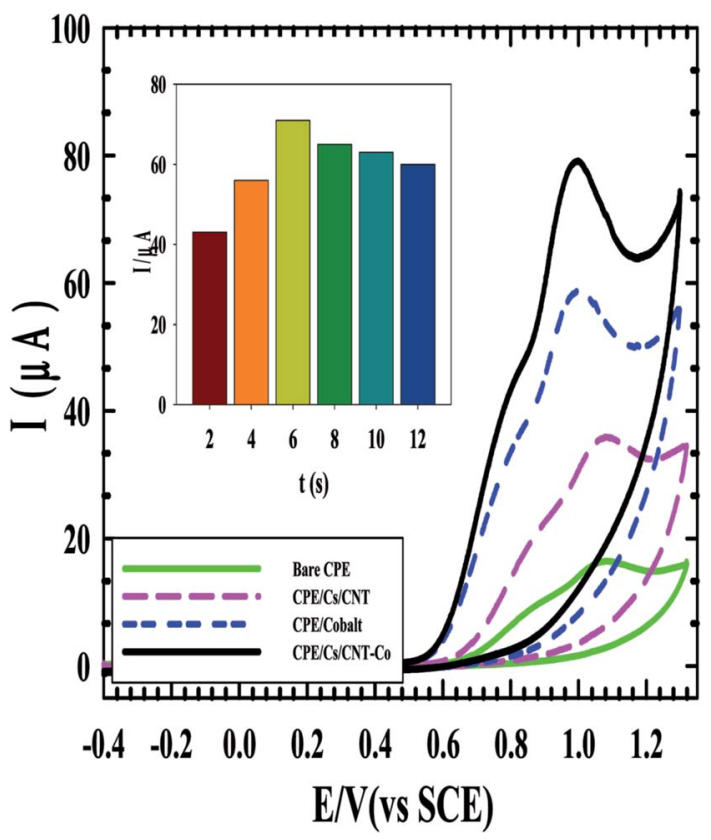

Fig. 1 Electrochemical oxidation responses of DAC in B-R buffer $\mathrm{pH}$ 2.0 using different electrodes surfaces. The inset: represents the effect of changing the electrodeposition time of Co nanoparticles on CPE/ Cs/CNT. distributed tubes (inset) providing more porosity, causing an enhancement in the effective surface area of CPE/Cs/CNT, so it could accumulate more CoNps on its surface. The SEM of CPE/ Cs/CNT-Co appears to be light, cloudy and spherical nanoparticles with diameters ranging from $40-80 \mathrm{~nm}$.

3.1.2.2. TEM. The TEM image of (A) CPE/Cs/CNT and (B) $\mathrm{CPE} / \mathrm{Cs} / \mathrm{CNT}-\mathrm{Co}$ was presented in Fig. 3. CPE/Cs/CNT shows $\mathrm{CNT}$ as a curly, bamboo like structure with diameter $\approx 25 \mathrm{~nm}$, while CPE/Cs/CNT-Co shows Co nanoparticles with average diameter $\approx 35 \mathrm{~nm}$.

3.1.2.3. EDX and elemental mapping. EDX data of CPE/Cs/ CNT-Co (Fig. 4) indicated the presence of Co, carbon and oxygen peaks. Also the elemental analysis confirmed that about $92 \%$ of $\mathrm{CPE} / \mathrm{Cs} / \mathrm{CNT}-\mathrm{Co}$ is coated by CoNps. The elemental mapping confirms that $\mathrm{O}, \mathrm{C}$ and $\mathrm{Co}$ are uniformly distributed across the whole mapped area on the sensor's surface.

3.1.3. Effect of $\mathbf{p H}$. The amount of current of $1.0 \mathrm{mM}$ DAC in $\mathrm{B}-\mathrm{R}$ buffer with different $\mathrm{pH}$ values was detected using cyclic voltammetry on CPE/Cs/CNT-Co surface (Fig. 5A). The best result was obtained at $\mathrm{pH}$ 2.0. The comparison of the influence of $\mathrm{pH}$ on the anodic peak current of DAC upon using bare CPE and CPE/Cs/CNT-Co was shown in Fig. 5B, the results show that in acidic $\mathrm{pH}$ values $(<6.0)$, higher currents appear due to the $\mathrm{p} K_{\mathrm{a}}$ value of DAC which is 6.09. It was also detected that the peak potentials shifted to less positive values with increasing $\mathrm{pH}$, leading to a linear regression equation expressed as $E_{\mathrm{p}}=$ $-0.0578 \mathrm{pH}-1.197(R=0.9993)$. The slope of $57 \mathrm{mV} \mathrm{pH}^{-1}$ indicates that the system obeys the Nernst equation. Also the Nyquist plots for CPE/Cs/CNT-Co at different $\mathrm{pH}$ values were shown in Fig. 5C with semicircles and lines, each corresponds to a charge transfer resistance and a diffusion process at both high and low frequencies, respectively. It is clear from Nyquist plot that the mechanism depends on diffusion and charge 

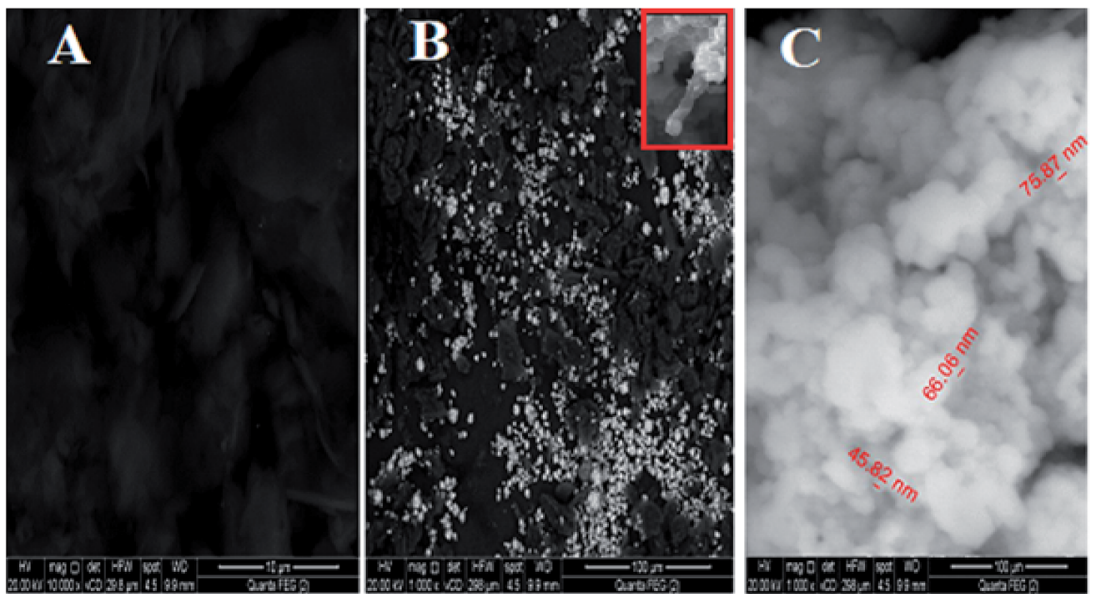

Fig. 2 The SEM of (A) bare CPE, (B) CPE/Cs/CNT and (C) CPE/Cs/CNT-Co.
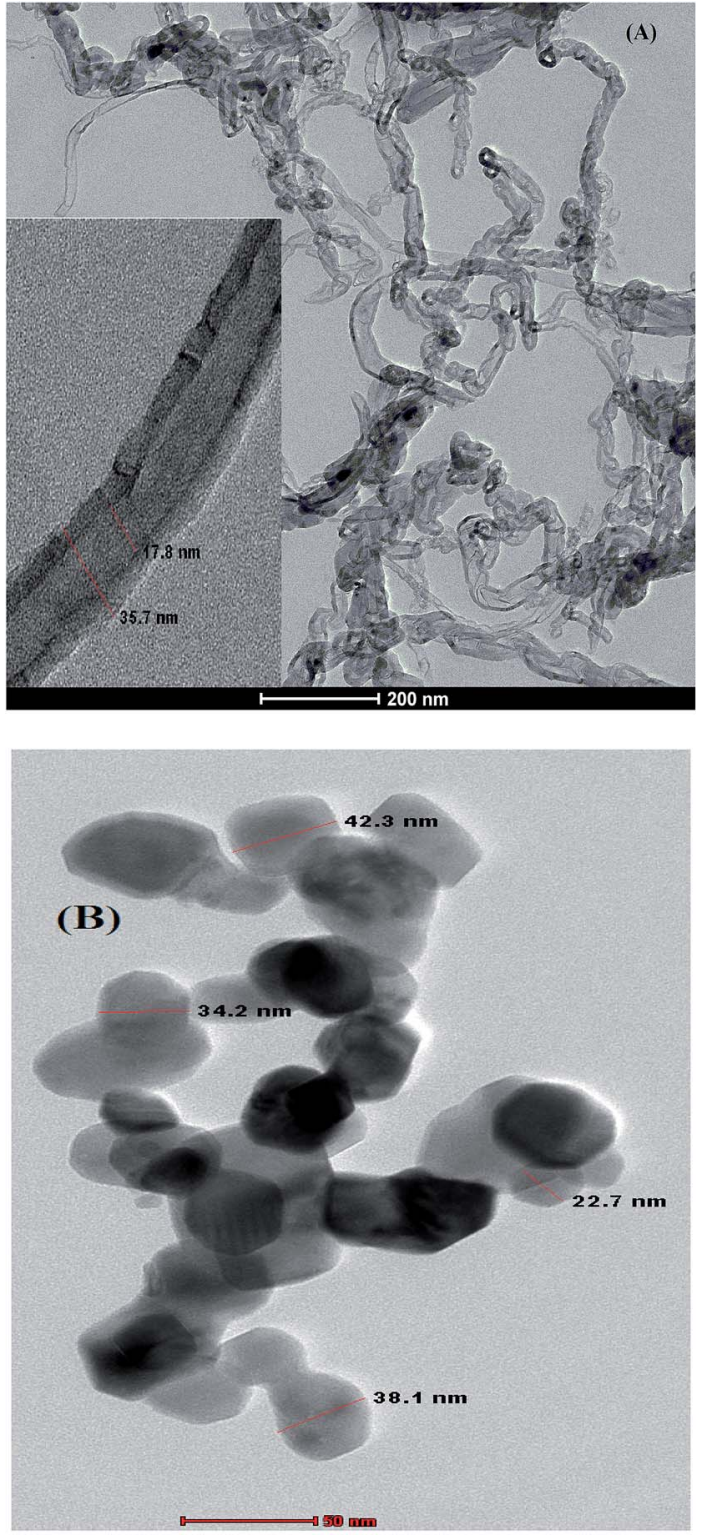

Fig. 3 TEM image of (A) CPE/Cs/CNT and (B) CPE/Cs/CNT-Co. transfer mechanism. The semicircle diameter and the impedance values increase with increasing $\mathrm{pH}$ values indicating a decrease in the conductivity ${ }^{\mathbf{2 1 , 2 2}}$ and giving the same order of the CVs results. ${ }^{22}$ The curves are simulated with one time constant model (Scheme 3). $R_{\mathrm{s}}$ is the solution resistance, $R$ is the charge transfer resistance and $W$ is Warburg impedance for diffusion. ${ }^{23,24}$ The Constant-Phase Element (CPE) of the capacitance is used instead of ideal capacitance owing to surface heterogeneity. ${ }^{25}$ Thus, the impedance value at bare electrode is $243 \mathrm{k} \Omega \mathrm{cm}^{2}$ compared to $111 \mathrm{k} \Omega \mathrm{cm}^{2}$ for the modified CPE/Cs/ CNT-Co electrode at $\mathrm{pH}$ 2.0.

3.1.4. Effect of scan rate. The influence of changing the scan rate over the range of $10-300 \mathrm{mV} \mathrm{s}^{-1}$ was studied to detect the diffusion and adsorption properties of $1.0 \mathrm{mM}$ DAC at CPE/ Cs/CNT-Co (Fig. 6). The positive shift in the peak potential approves that the oxidation process of DAC is irreversible. The plot of peak current versus square root of scan rate was constructed (inset A) and a straight line was obtained fulfilling the equation $I_{\mathrm{p}}(\mu \mathrm{A})=9.872 \sqrt{ } \nu-14.48 ; R=0.9916$. This relationship indicates that diffusion mechanisms are involved in the electrochemical reaction. Also a plot of log peak current versus $\log$ scan rate was performed giving a linear relationship with a slope of 0.572 (inset B), suggesting that DAC oxidation on CPE/ Cs/CNT-Co is primarily controlled by diffusion with some adsorption support, as observed from impedance data. Thus, CV data ensure well EIS data.

The electro-active surface area of bare CPE and CPE/Cs/CNTCo were achieved by $\mathrm{CV}$ technique, using $1.0 \mathrm{mM} \mathrm{K}_{3} \mathrm{Fe}(\mathrm{CN})_{6}$ based on the Randles-Sevcik equation: ${ }^{2,27}$

$$
I_{\mathrm{p}}=2.69 \times 10^{5} n^{3 / 2} A D^{1 / 2} \nu^{1 / 2} C
$$

For $\mathrm{K}_{3} \mathrm{Fe}(\mathrm{CN})_{6}, n=1$ and $D=7.6 \times 10^{-6} \mathrm{~cm}^{2} \mathrm{~s}^{-1}$, the area were evaluated to be $0.072 \mathrm{~cm}^{2}$ and 0.217 , for bare CPE and CPE/ Cs/CNT-Co, respectively. Thus after modification the surface area of the electrodes was considerably increased. These areas were used to calculate the diffusion coefficient of $1.0 \mathrm{mM}$ DAC at $100 \mathrm{mV} \mathrm{s}^{-1}$ which increases from $9.37 \times 10^{-8} \mathrm{~cm}^{2} \mathrm{~s}^{-1}$ in case of using bare CPE to $2.24 \times 10^{-7} \mathrm{~cm}^{2} \mathrm{~s}^{-1}$ after the functionalization of CPE/Cs/CNT surface with Co nanoparticles. 

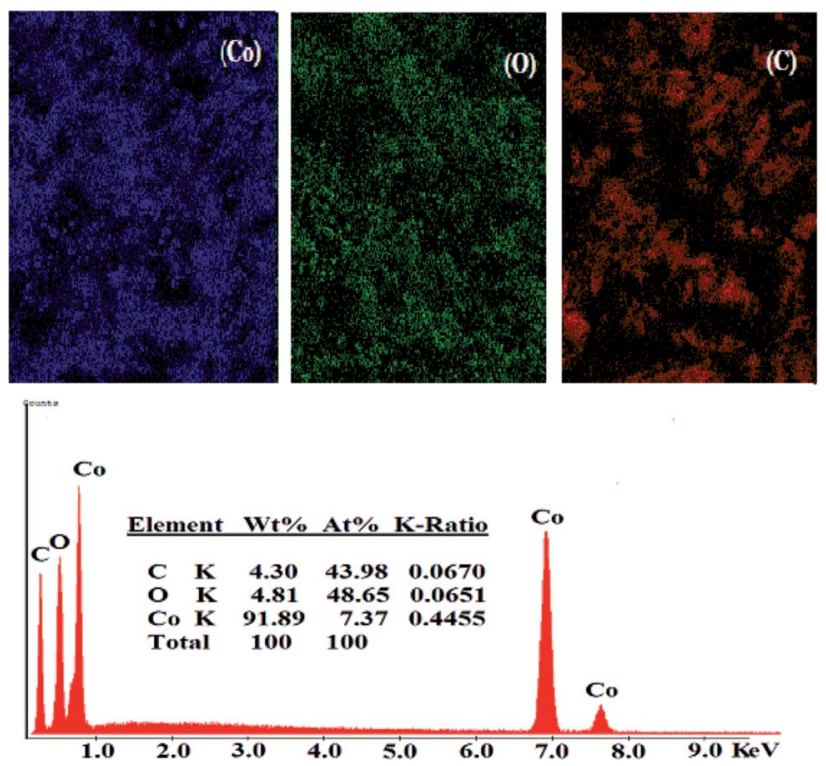

Fig. 4 The elemental mapping and the EDX with the elemental analysis data of CPE/Cs/CNT-Co surface.

To find out the surface coverage of the adsorbed molecule; eqn (2) was used. ${ }^{28}$

$$
I_{\mathrm{p}}=\left(n^{2} F^{2} A \Gamma \nu / 4 R T\right)
$$

The surface coverage of the adsorbed molecule $(T)$ is a very important parameter that indicates the behavior of the sensor, and they were found to be $1.35 \times 10^{-10} \mathrm{~mol} \mathrm{~cm}^{-2}$ and $2.278 \times$ $10^{-10} \mathrm{~mol} \mathrm{~cm}^{-2}$ for bare CPE and CPE/Cs/CNT-Co, respectively. The results show that the catalytic activity of DAC increases in the presence of Cs/CNT and Co nanoparticles. Also the determination of the sensor dynamics parameters $(\alpha$ and $n)$ is essential to determine the proposed mechanism of DAC. The relationship between $E_{\mathrm{p}}$ and $\ln \nu$ (inset $\mathrm{C}$ ) is given by Laviron equation: ${ }^{14}$

$$
E_{\mathrm{p}}=E_{0}+[R T /(\alpha n F)] \ln \left[\left(R T k_{\mathrm{s}}\right) /(\alpha n F)\right]+[R T /(\alpha n F)] \ln \nu
$$

where $\alpha$ is the electron transfer coefficient, $k_{\mathrm{s}}$ is the standard rate constant of the surface reaction and $E_{0}$ is the formal potential obtained from the relation between $E_{\mathrm{p}}$ and $\nu$ by extrapolating to the vertical axis at $\nu=0$ and it was found to be $0.965 \mathrm{~V}$ (inset D), $\alpha n$ can be calculated from the slope of $E_{\mathrm{p}} v s$. $\ln v$. In this system, the slope is $0.048, \alpha n$ was calculated to be 1.87. Since for a totally irreversible electron transfer, $\alpha$ assumed as 0.5 , then $n$ was calculated to be 3.74 which indicated that four electrons were involved in the oxidation of DAC. The value of $k_{\mathrm{s}}$ can be calculated from the intercept of Laviron equation and was found to be $2.44 \times 10^{3} \mathrm{~s}^{-1}$.

\subsection{Chronoamperometry measurements}

Chronoamperometry data measured for DAC at a constant potential of $+990 v s$. SCE are shown in Fig. 7. The calibration curve has been derived at early times of 2 seconds which gives

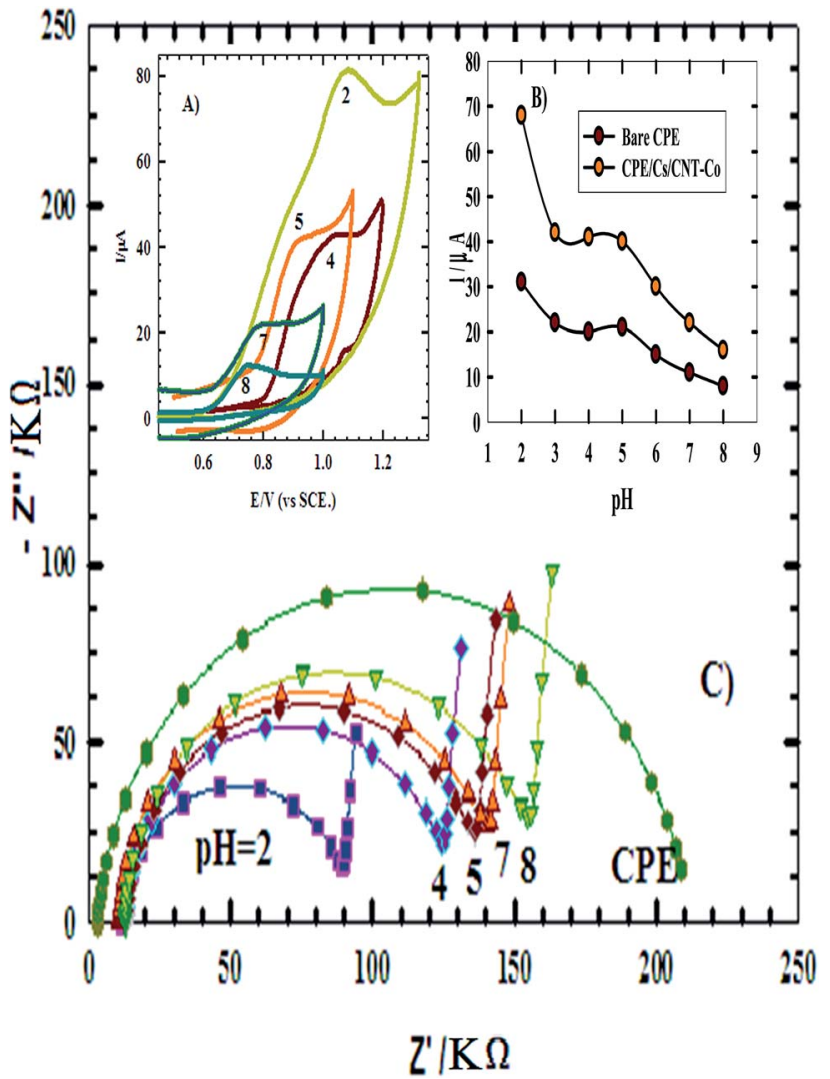

Fig. 5 (A) Cyclic voltammograms of $1.0 \mathrm{mM}$ DAC in B-R buffer at different $\mathrm{pH}$ values, with scan rate $100 \mathrm{mV} \mathrm{s}^{-1}$ recorded at CPE/Cs/ CNT-Co. (B) Graph of current response of DAC at different $\mathrm{pH}$ values using bare CPE and CPE/Cs/CNT-Co. (C) Nyquist plots for DAC using bare $\mathrm{CPE}$ and $\mathrm{CPE} / \mathrm{Cs} / \mathrm{CNT}-\mathrm{Co}$ in $\mathrm{B}-\mathrm{R}$ buffer with different $\mathrm{pH}$ values.

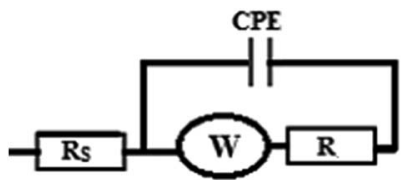

Scheme 3

the best correlation coefficient of $0.997 .^{29,30}$ The linear equation is $I_{\mathrm{p}}(\mu \mathrm{A})=35 C+59$. Thus, the response time is 2 seconds and the recovery is 17 seconds at which the current becomes stable. This response time is lower than that observed by Arora et al. ${ }^{31}$ $(60 \mathrm{~s})$, Ali and Mohamed $^{32}(5 \mathrm{~s})$. Du et al. ${ }^{33}$ with response time of $7 \mathrm{~s}$ and Tang et al..$^{34}$ biosensor with a response time of less than $15 \mathrm{~s}$.

\subsection{Characterization and linearity of CPE/Cs/CNT-Co}

Quantitative determination based on the linear relationship between the oxidation current and the concentration of DAC from $1.0 \times 10^{-9} \mathrm{M}$ to $1.2 \times 10^{-5} \mathrm{M}$ (Fig. 8) at CPE/Cs/CNT-Co was studied using DPV at scan rate $10 \mathrm{mV} \mathrm{s}^{-1}$ (inset A). The parameters of the calibration plots are summarized in Table 1. The limit of detection (LOD) and the limit of quantification (LOQ) were calculated using the following equations: $\mathrm{LOD}=3 \mathrm{~S} /$ 


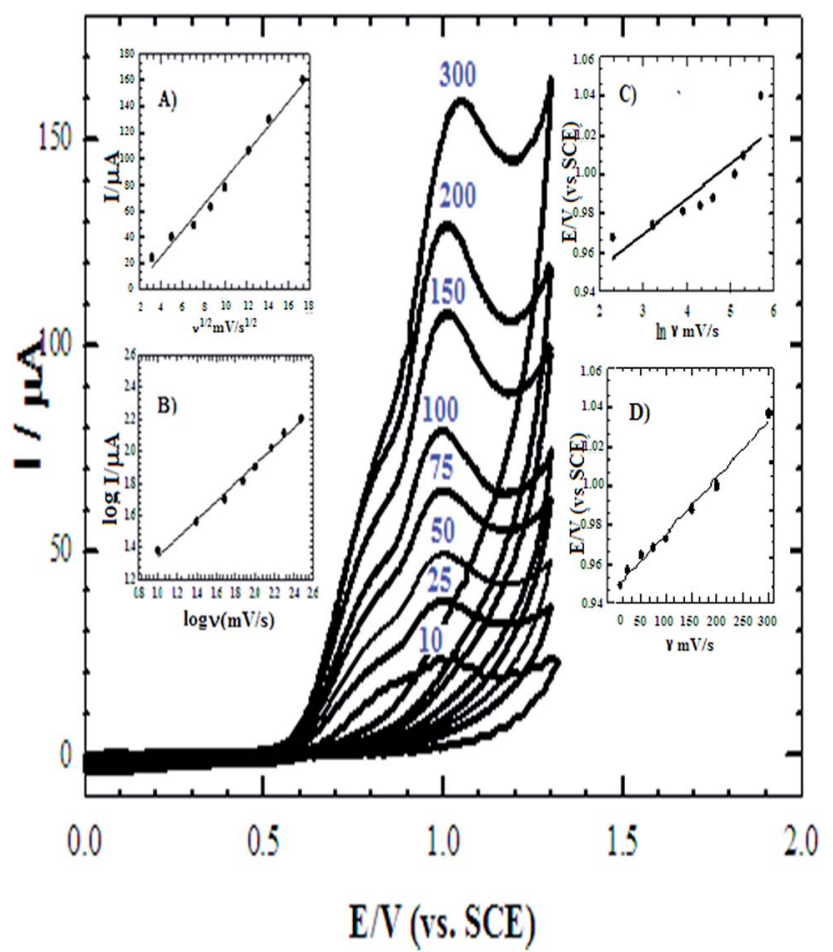

Fig. 6 Cyclic voltammograms (CV) of $1.0 \mathrm{mM} \mathrm{DAC}$ at CPE/Cs/CNT-Co in B-R buffer $\mathrm{pH} 2.0$ at: 10, 25, 50, 75, 100, 150, 200 and $300 \mathrm{mV} \mathrm{s}^{-1}$. Inset (A) plot of the anodic peak current values versus square root of scan rate. Inset (B) plot of log the anodic peak current versus log the scan rate. Inset (C) plot of the anodic peak potential values versus In scan rate. Inset (D) plot of the anodic peak potential values versus scan rate.

$m$ and LOQ $=10 \mathrm{~S} / \mathrm{m} \cdot{ }^{35}$ Where $S$ is the standard deviation and $m$ is the slope of the calibration curve and was found to be $8.82 \times$ $10^{-10} \mathrm{M}$ and $2.94 \times 10^{-9} \mathrm{M}$, respectively. Also, the effect of concentration was studied using impedance spectroscopy (inset B). The results showed that as the concentration increases, impedance value decreases, where the ionic/electronic charge transfer resistance shows noticeable decrease in values indicating less electronic resistance and more conductivity. The optimized procedure has also been well applied for the determination of DAC in spiked serum and urine samples. The obtained validation data are given in Table 1 and compared with that obtained from the raw material.

\subsection{Effect of some biological interferences and co- administered drugs}

Ascorbic acid (AA) and uric acid (UA) are the most important interferences that coexist in the extracellular fluid of the central nervous system. To check the sensitivity of the method, the electrocatalytic oxidation of DAC in the presence of $1.0 \mathrm{mM}$ AA and 1.0 mM UA was investigated in urine sample at the surface of bare CPE and CPE/Cs/Co using DPV. Fig. 9A shows that weak oxidation responses were detected at bare CPE with some overlapping between AA and UA while upon using CPE/Cs/Co the oxidation peaks of DAC (at $800 \mathrm{mV}$ ), AA (at $390 \mathrm{mV}$ ) and UA $(266 \mathrm{mV})$ were higher, sharper and well separated. DAC may also interact with anticonvulsants, antimycobacterial agents

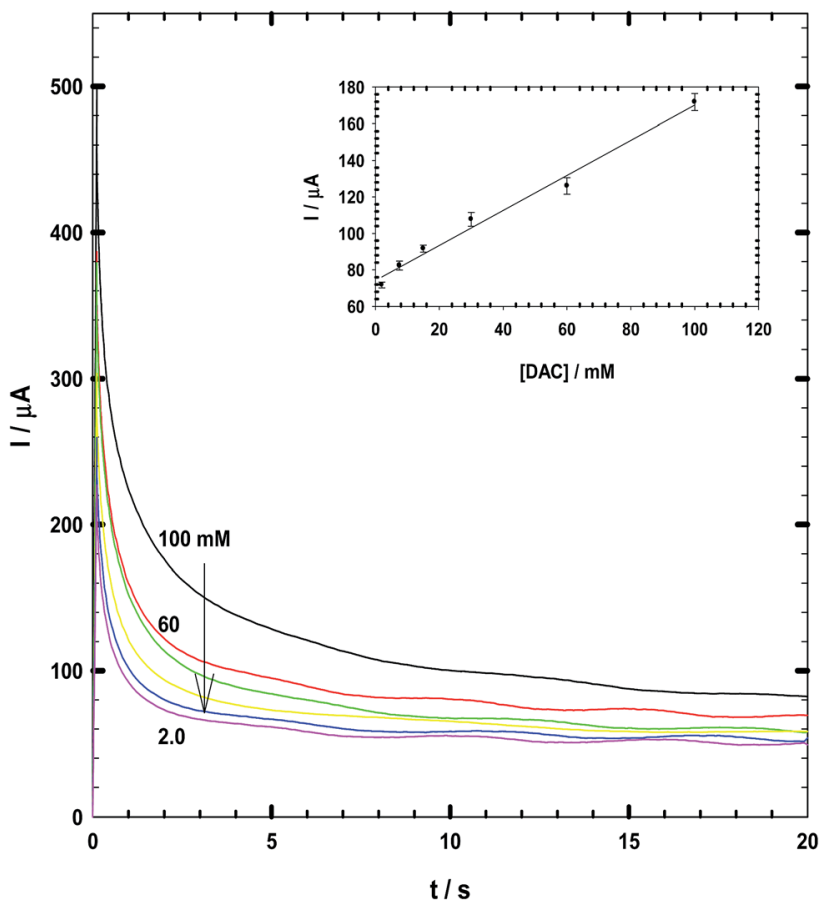

Fig. 7 Chronoamperometry diagram for 1 mM DAC in $\mathrm{B}-\mathrm{R}$ buffer $\mathrm{pH} 2$.

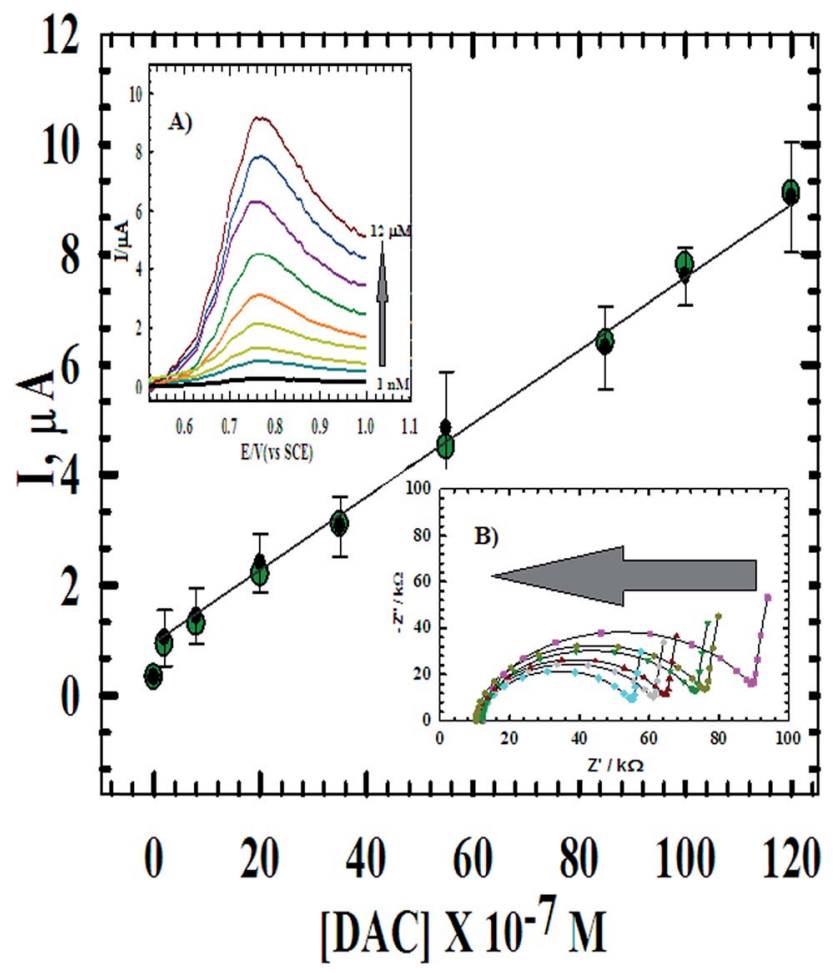

Fig. 8 The calibration curve of DAC at CPE/Cs/CNT-Co. Inset (A) DPV of the successive additions of DAC in $B-R$ buffer and $10 \mathrm{mV} \mathrm{s}^{-1}$. Inset (B) the Nyquist plot of the successive additions of DAC.

and other antiviral drugs. To check the sensitivity of the method, the electrocatalytic oxidation of DAC in the presence of entecavir (ENT) (an antiviral drugs used in the treatment of 
Table 1 Regression data of the linear ranges by DPV using CPE/Cs/CNT-Co for quantitative determination of DAC in raw material, urine sample and serum sample

\begin{tabular}{|c|c|c|c|}
\hline Parameters & Raw material & Urine sample & Serum sample \\
\hline Linearity (M) & $1.0 \times 10^{-9}$ to $1.2 \times 10^{-5}$ & $6 \times 10^{-9}$ to $1.1 \times 10^{-5}$ & $9 \times 10^{-9}$ to $1.1 \times 10^{-5}$ \\
\hline LOD (M) & $8.82 \times 10^{-10}$ & $1.52 \times 10^{-9}$ & $7.07 \times 10^{-9}$ \\
\hline LOQ (M) & $2.93 \times 10^{-10}$ & $5.06 \times 10^{-9}$ & $2.35 \times 10^{-8}$ \\
\hline Slope & 0.0704 & 0.0841 & 0.0611 \\
\hline SE of slope & 0.0088 & 0.0038 & 0.0055 \\
\hline Intercept & 0.6320 & 0.9110 & 1.0131 \\
\hline SE of intercept & 0.0017 & 0.0744 & 0.0624 \\
\hline Correlation coefficient $(r)$ & 0.9997 & 0.9993 & 0.9990 \\
\hline
\end{tabular}
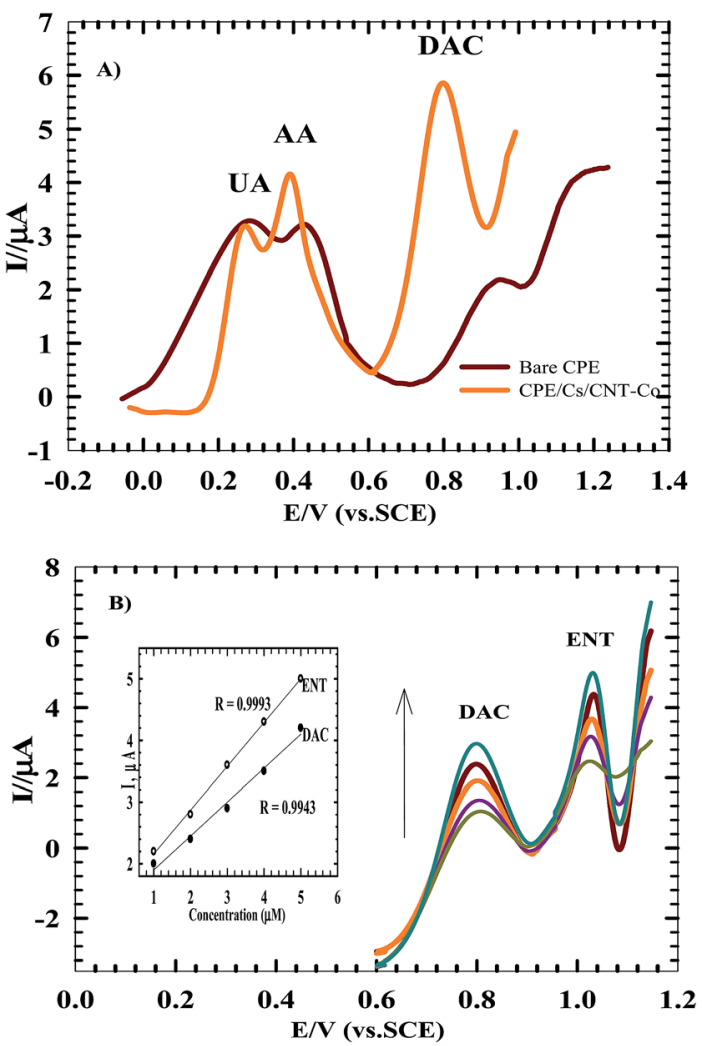

Fig. 9 (A) DPV of $\mathrm{B}-\mathrm{R}$ buffer solution $(\mathrm{pH} 2.0)$ containing $D A C$ with $A A$ and UA monitored at bare CPE and CPE/CS/CNT-Co. (B) DPV of B-R buffer solution ( $\mathrm{pH} 2.0$ ) containing DAC and ENT by varying their concentrations from 1 to $5 \mu \mathrm{M}$ at CPE/Cs/CNT-Co. Inset: electrocatalytic peak current versus DAC and ENT concentration.

chronic hepatitis B in adults with compensated liver disease of active viral replication), is an important issue to be studied since both drugs are co administered by patients who suffer from virus B and $\mathrm{C}$. The simultaneous determination studied in B-R buffer using CPE/Cs/CNT-Co exhibits two anodic peaks for $\mathrm{DAC}$ and ENT at $0.79 \mathrm{~V}$ and $1.12 \mathrm{~V}$, respectively with good peak separation of $0.33 \mathrm{~V}$. The current response increased linearly with increasing of concentrations of both compounds (Fig. 9B) obeying the following equations:

$$
I_{\mathrm{p}}(\mu \mathrm{A})=0.550 C(\mu \mathrm{M})-1.350, \text { for DAC }
$$

$$
I_{\mathrm{p}}(\mu \mathrm{A})=0.710 C(\mu \mathrm{M})-1.450, \text { for ENT }
$$

These results show the high selectivity of the proposed method for the determination of DAC.

\subsection{Stability of repeated CVs}

Fig. 10 A shows the repeated CVs for $1 \mathrm{mM}$ DAC in B-R buffer $(\mathrm{pH}=2.0)$ at $\mathrm{CPE} / \mathrm{Cs} / \mathrm{CNT}-\mathrm{Co}$ for 50 cycles. The decrease of the peak current from the first to the last cycle was about $10 \%$. Also an impedance spectroscopy experiment was performed at the first cycle and after 50 cycles (Fig. 10B). It was found that the curve shape doesn't change. This ensures that the mechanism is the same which depends on both charge transfer and diffusion processes. Impedance value from the first to 50 cycles increases with a small value indicating a low decrease in current value. FT-IR analyses were performed for both $\mathrm{CPE} / \mathrm{Cs} / \mathrm{CNT}-\mathrm{Co}$ and $\mathrm{CPE} / \mathrm{Cs} / \mathrm{CNT}$ electrodes in absence and presence of DAC after 50 cycles. For CPE/Cs/CNT, MWCNTs absorption band at $1615 \mathrm{~cm}^{-1}$ is due to the stretching of $\mathrm{C}=\mathrm{C}$ bonds and the peak at $2885 \mathrm{~cm}^{-1}$ is due to the stretching of $\mathrm{C}-\mathrm{H}$ bonds. Cs shows absorption peaks at $3427 \mathrm{~cm}^{-1}$ for $-\mathrm{OH}$ group and $2885 \mathrm{~cm}^{-1}$ for $\mathrm{CH}_{3}$ groups. The interactions between DAC with MWCNT and chitosan occurs via hydrogen bonds formation between the $-\mathrm{OH}$ of $\mathrm{Cs}$ and $\mathrm{C}=\mathrm{C}$ in CNT with $-\mathrm{NH}$ in DAC, allowing the formation of electrostatic interactions (Fig. 10C). A strong IR changes were also observed in FT-IR charts in case of CPE/Cs/ CNT-Co due to the presence of Co nanoparticles that appears as doublet at $567 \mathrm{~cm}^{-1}$ and $646 \mathrm{~cm}^{-1}$ indicating a very strong interaction between the electrode surfaces and DAC (Fig. 10D).

\subsection{Analytical application and robustness}

For the purpose of the practical applicability of the proposed method and the modified sensor, DPV method was effectively applied for the investigation of DAC in five Daktavira tablets without any sample extraction and after dilution to obtain 1.0 mM DAC solution. The validity of the proposed method was evaluated by the standard addition technique and the accuracy has been determined giving satisfactory recoveries from 98.40 to $101.6 \%$ and relative standard deviation from 0.03 to $0.43 \%$ reveling that the excipient presented in tablet does not interfere with the active ingredient. The results suggested that CPE/Cs/ CNT-Co has high reproducibility and would be useful sensor for quantitative analysis of DAC in pharmaceutical 

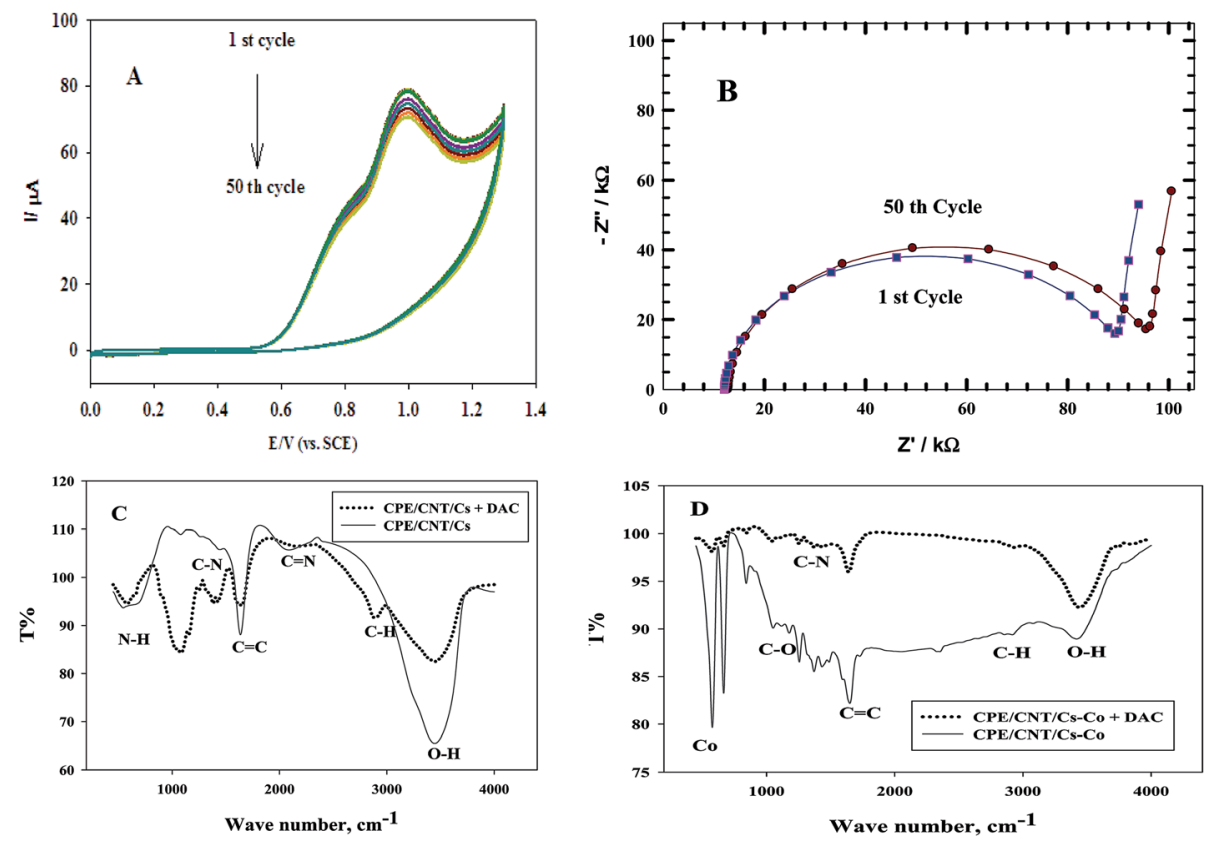

Fig. 10 (A) Repeated CVs of $1 \mathrm{mM} \mathrm{DAC}$ in $\mathrm{B}-\mathrm{R}$ buffer $(\mathrm{pH}=2.0$ ) at CPE/Cs/CNT-Co for 50 times. (B) Impedance spectroscopy of the first and the $50^{\text {th }}$ cycles of $1 \mathrm{mM}$ DAC in $\mathrm{B}-\mathrm{R}$ buffer $(\mathrm{pH}=2.0)$ at CPE/Cs/CNT-Co (C) FT-IR spectrum of CPE/Cs/CNT in the presence and absence of DAC (D) FT-IR spectrum of CPE/Cs/CNT-Co in the presence and absence of DAC.

formulations. The robustness was examined by evaluating the variation of $\mathrm{pH}=2.5$ instead of $\mathrm{pH}=2.0$. The obtained results indicated that the proposed procedures for DAC assay can be considered robust.

\section{Conclusion}

This work establishes the ability of a new CPE sensor for the electrochemical determination of the antiviral drug DAC in pharmaceutical formulations, biological samples and in the presence of the co-administered drug ENT. Some parameters such as the diffusion coefficient, surface coverage and the number of electrons involved in the oxidation reaction were calculated. The results showed that the method was sensitive, precise and selective with no significant effects of the excipients background. Experimental results specify a linear relation between DAC peak current and its concentration in the range from $1.0 \mathrm{nM}$ to $12 \mu \mathrm{M}$, leading to a detection limit of $8.82 \times$ $10^{-10} \mathrm{M}$. Finally, this novel sensor was successfully used to determine DAC in human biological fluids such as urine and blood serum and in pharmaceutical formulations. So this sensor exhibited attractive specific capacitance and long life time, which will supply a new insight for various applications in the future.

\section{References}

1 L. B. Jerez, U. García-Pérez, P. Zambrano-Robledo and J. Hernández-Moreira, Carbon paste electrode modified with $\mathrm{BiVO}_{4}$ to sense metformin, Int. J. Electrochem. Sci., 2014, 9, 4643-4652.
2 T. Haak, Combination of linagliptin and metformin for the treatment of patients with type 2 diabetes, Clinical medicine insights, J. Diabetes Endocrinol., 2015, 8, 1.

3 J. J. O'Dea, A. Ribes and J. G. Osteryoung, Square-wave voltammetry applied to the totally irreversible reduction of adsorbate, J. Electroanal. Chem., 1993, 345, 287-301.

4 N. Padmaja and G. Veerabhadram, Development and Validation of Analytical Method for Simultaneous Estimation of Empagliflozin and Linagliptin in Bulk Drugs and Combined Dosage Forms Using UV-Visible Spectroscopy, Pharm. Lett., 2015, 7, 306-312.

5 L. R. Badugu, A Validated RP-HPLC method for the determination of Linagliptin, Am. J. PharmTech Res., 2012, 2, 463-470.

6 S. Banik, P. Karmakar and M. A. H. Miah, Development and Validation of a UV-Spectrophotometric Method for Determination of Vildagliptin and Linagliptin in Bulk and Pharmaceutical Dosage Forms, Bangladesh Pharm. J., 2015, 18, 163-168.

7 L. Özcan and Y. Şahin, Determination of paracetamol based on electropolymerized-molecularly imprinted polypyrrole modified pencil graphite electrode, Sens. Actuators, B, 2007, 127, 362-369.

8 R. N. Goyal, V. K. Gupta and S. Chatterjee, Voltammetric biosensors for the determination of paracetamol at carbon nanotube modified pyrolytic graphite electrode, Sens. Actuators, B, 2010, 149, 252-258.

9 M. Ansari, S. Kazemi, M. A. Khalilzadeh, H. Karimi-Maleh and M. P. Zanousi, Sensitive and stable voltammetric measurements of norepinephrine at ionic liquid-carbon nanotubes paste electrodes, Int. J. Electrochem. Sci., 2013, 8, 1938-1948. 
10 M. Zidan, W. T. Tan, Z. Zainal, A. H. Abdullah and J. K. Goh, Electrocatalytic oxidation of ascorbic acid mediated by lithium doped microparticles $\mathrm{Bi}_{2} \mathrm{O}_{3} / \mathrm{MWCNT}$ modified glassy carbon electrode, Int. J. Electrochem. Sci., 2010, 5, 501-508.

11 C. J. Bailey and R. C. Turner, Metformin, N. Engl. J. Med., 1996, 334, 574-579.

12 D. Pletcher, Electrocatalysis: present and future, J. Appl. Electrochem., 1984, 14, 403-415.

13 Y. B. Vassilyev, O. Khazova and N. Nikolaeva, Kinetics and mechanism of glucose electrooxidation on different electrode-catalysts: Part I. Adsorption and oxidation on platinum, J. Electroanal. Chem. Interfacial Electrochem., 1985, 196, 105-125.

14 Y. Shen, J. Tang, Z. Nie, Y. Wang, Y. Ren and L. Zuo, Preparation and application of magnetic $\mathrm{Fe}_{3} \mathrm{O}_{4}$ nanoparticles for wastewater purification, Sep. Purif. Technol., 2009, 68, 312-319.

15 M. R. Rezk, E. R. Bendas, E. B. Basalious and I. A. Karim, Development and validation of sensitive and rapid UPLCMS/MS method for quantitative determination of daclatasvir in human plasma: Application to a bioequivalence study, $J$. Pharm. Biomed. Anal., 2016, 128, 61-66.

16 G. Srinivasu, K. N. Kumar, C. Thirupathi, C. L. Narayana and C. P. Murthy, Development and Validation of the Chiral HPLC Method for Daclatasvir in Gradient Elution Mode on Amylose-Based Immobilized Chiral Stationary Phase, Chromatographia, 2016, 79, 1457-1467.

17 V. A. Chakravarthy and B. Sailaja, Method Development and Validation of Assay and Dissolution Methods for The Estimation of Daclatasvir in Tablet Dosage Forms by Reverse Phase HPLC, European Journal of Pharmaceutical and Medical Research, 2016, 3, 356-364.

18 N. Jawahara, E. Surendraa and K. R. Krishnaa, A Review on Carbon Nanotubes: A Novel drug Carrier for Targeting to Cancer Cells, J. Pharm. Sci. Res., 2015, 7, 141-154.

19 I. Streeter, G. G. Wildgoose, L. Shao and R. G. Compton, Cyclic voltammetry on electrode surfaces covered with porous layers: an analysis of electron transfer kinetics at single-walled carbon nanotube modified electrodes, Sens. Actuators, B, 2008, 133, 462-466.

20 S. Shahrokhian, E. Jokar and M. Ghalkhani, Electrochemical determination of piroxicam on the surface of pyrolytic graphite electrode modified with a film of carbon nanoparticle-chitosan, Microchim. Acta, 2010, 170, 141-146.

21 B. Polyak and G. Friedman, Magnetic targeting for sitespecific drug delivery: applications and clinical potential, Expert Opin. Drug Delivery, 2009, 6, 53-70.

22 A. Jalilian, A. Panahifar, M. Mahmoudi, M. Akhlaghi and A. Simchi, Preparation and biological evaluation of [67Ga]labeled-superparamagnetic nanoparticles in normal rats, Radiochim. Acta, 2009, 97, 51-56.
23 F. E.-T. Heakal and A. Fekry, Experimental and theoretical study of uracil and adenine inhibitors in $\mathrm{Sn}-\mathrm{Ag}$ alloy/nitric acid corroding system, J. Electrochem. Soc., 2008, 155, C534-C542.

24 A. Fekry, Impedance and hydrogen evolution studies on magnesium alloy in oxalic acid solution containing different anions, Int. J. Hydrogen Energy, 2010, 35, 1294512951.

25 A. J. Bard, L. R. Faulkner, J. Leddy and C. G. Zoski, Electrochemical methods: fundamentals and applications, Wiley, New York, 1980.

26 N. F. Atta, A. Galal, F. M. Abu-Attia and S. M. Azab, Characterization and electrochemical investigations of micellar/drug interactions, Electrochim. Acta, 2011, 56, 2510-2517.

27 N. F. Atta, A. Galal and S. M. Azab, Electrochemical determination of neurotransmitters using gold nanoparticles on nafion/carbon paste modified electrode, J. Electrochem. Soc., 2012, 159, H765-H771.

28 A. M. Prodan, S. L. Iconaru, C. M. Chifiriuc, C. Bleotu, C. S. Ciobanu, M. Motelica-Heino, S. Sizaret and D. Predoi, Magnetic properties and biological activity evaluation of iron oxide nanoparticles, J. Nanomater., 2013, 2013, 893970.

29 B. Hemmateenejad, A. Safavi and F. Honarasa, Deriving calibration curves at early times of chronoamperograms using the chemometrically resolved net faradaic current, J. Electroanal. Chem., 2015, 755, 221-227.

30 A. M. Fekry, A new simple electrochemical Moxifloxacin Hydrochloride sensor built on carbon paste modified with silver nanoparticles, Biosens. Bioelectron., 2017, 87, 10651070.

31 K. Arora, M. Choudhary and B. D. Malhotra, Enhancing performance of uricase using multiwalled carbon nanotube doped polyaniline, Appl. Biochem. Biotechnol., 2014, 174, 1174-1187.

32 T. A. Ali and G. G. Mohamed, Determination of Mn (II) ion by a modified carbon paste electrode based on multi-walled carbon nanotubes (MWCNTs) in different water samples, Sens. Actuators, B, 2014, 202, 699-707.

33 X. Du, Z. Miao, D. Zhang, Y. Fang, M. Ma and Q. Chen, Facile synthesis of $\beta$-lactoglobulin-functionalized multi-wall carbon nanotubes and gold nanoparticles on glassy carbon electrode for electrochemical sensing, Biosens. Bioelectron., 2014, 62, 73-78.

$34 \mathrm{~W}$. Tang, L. Li, L. Wu, J. Gong and X. Zeng, Glucose biosensor based on a glassy carbon electrode modified with polythionine and multiwalled carbon nanotubes, PLOS One, 2014, 9, e95030.

35 N. F. Atta, A. Galal and S. M. Azab, Gold Nanoparticles Modified Electrode for the Determination of an Antihypertensive Drug, Electroanalysis, 2012, 24, 1431-1440. 